\title{
Time for the endocrinologists to expand their awareness of and contribution to the diagnosis and management of encephalopathy associated with autoimmune thyroid disease
}

\author{
Gianluca Tamagno, Gadintshware Gaoatswe \\ Department of Endocrinology \& Diabetes Mellitus, St Vincent's University Hospital, University College Dublin, Ireland
}

\begin{abstract}
Encephalopathy associated with autoimmune thyroid disease is a rare condition presenting in the setting of autoimmune thyroid disease and characterized by unspecific neurological and/ or psychiatric symptoms. Bearing in mind the currently prevailing lack of consensus on the most appropriate nomenclature and diagnostic criteria for this condition and the implications that this lack undeniably has on clinical practice, it is obvious that an international and multidisciplinary agreement among clinicians should arrive at the most appropriate definition and terminology of encephalopathy occurring in patients with autoimmune thyroid disease. Concomitantly, efforts must be made to uncover the pathogenetic link between thyroid autoimmunity and the occurrence of encephalopathy.
\end{abstract}

Key Words: Autoimmune thyroid disease, Encephalopathy, Graves' disease, Hashimoto's thyroiditis

Encephalopathy associated with autoimmune thyroid disease (EAATD), first described in 1966 by Brain and colleagues, ${ }^{1}$ is a rare condition presenting in the setting of autoimmune thyroid disease and characterized by neurological and/or psychiatric symptoms. ${ }^{1,2}$ The majority of EAATD cases are associated with Hashimoto's thyroiditis (HT), but recently EAATD has been shown to also occur in patients with Graves' disease (GD). ${ }^{3}$

Address for correspondence:

Dr Gianluca Tamagno, Department of Endocrinology \& Diabetes Mellitus, St Vincent's University Hospital University College Dublin, 4 Elm Park, Dublin 4, Ireland Tel.: +353 1221 4407, E-mail: gianlucatamagno@tiscali.it Received 24-08-10, Revised 30-10-10, Accepted 10-11-10
The terminology in the medical literature has varied, some authors using the traditional term Hashimoto's encephalopathy (HE) based on the observation that the majority of cases have been identified in patients with HT. Recently, the term EAATD ${ }^{4}$ as well as steroid responsive encephalopathy associated with autoimmune thyroiditis (SREAT) ${ }^{5}$ have been suggested in order to define more precisely the nosological picture and to highlight the concept of steroid responsiveness. In fact, steroid responsiveness has also been proposed as one of the possible criteria for making the diagnosis of EAATD. ${ }^{6,7}$ Owing to the implications that this lack of precise definition can have in clinical practice, there is an obvious need for international consensus among clinicians on 
the most appropriate terminology and definition of encephalopathy occurring in patients with autoimmune thyroid disease. ${ }^{8}$ Moreover, the aforementioned discrepancies in nomenclature makes it difficult to achieve a homogeneous literature on disease characteristics, a situation further aggravated by the rarity of EAATD. The most appropriate approach would be for the terminology to be based on the pathogenetic mechanism which, however, is not currently feasible as the pathogenesis of EAATD has not yet been precisely elucidated.

From a clinical point of view and based on the present knowledge, a designation such as EAATD appears to be appropriate as any autoimmune thyroid disease can be associated with encephalopathy. ${ }^{3}$ Such a terminology includes patients with either HT or GD. Although Castillo and colleagues included steroid responsiveness in their case series in defining encephalopathy occurring in patients with autoimmune thyroiditis, ${ }^{6}$ not all cases of EAATD respond to treatment with steroids, resulting in a not negligible number of non-responders or poorly responsive patients. ${ }^{9}$ Furthermore, the therapeutic response to steroids can occur in a wide range of autoimmune and inflammatory neurological conditions characterized by a picture of encephalopathy, making the requirement of steroid responsiveness not really specific for EAATD.

Since EAATD is a rare condition and, as mentioned above, many discrepancies exist in the literature, this compounded by the absence of international and multidisciplinary consensus, the diagnostic pathway is not as yet clearly defined. ${ }^{7,10}$ Obviously, this lack of agreement affects the evaluation of the actual prevalence of EAATD in patients with autoimmune thyroid disease.

The clinical presentation of EAATD is characterized by protean and non-specific symptoms, while distinctive neuroimaging or electroencephalographic findings have not been identified. Although anti-thyroid antibodies, such as anti-thyroperoxidase antibodies, are usually present in EAATD patients, a specific immunological marker for this condition has yet to be identified. ${ }^{8}$ Together with the subsistence of thyroid autoimmunity, the exclusion of other possible causes of encephalopathy still remain the cornerstone for diagnosing EAATD. ${ }^{8}$ We believe that there is a need for a consensus among endocrinologists, neurologists, psychiatrists, immunologists, pathologists, and neuroradiologists on the definition and the diagnostic criteria of EAATD.

Although endocrinologists are the main caregivers of patients with autoimmune thyroid disease, neurologists have had the lion's share in describing and reporting patients with EAATD. There is in fact a regrettable paucity of papers about EAATD published in endocrinology journals, with approximately $85 \%$ of the papers being published in neurology and general medicine journals. An important issue in this situation is the fact that, since EAATD pathogenesis is not as yet known and the link between autoimmune thyroid disease and encephalopathy has not been fully elucidated, considerable skepticism prevails among some endocrinologists as to the actual existence of EAATD. In other words, the current lack of certainty does not conduce to their perceiving the two pathologic manifestations, namely autoimmune thyroid disease and encephalopathy, as related clinical events. However, we strongly feel that this point should now represent an issue on which we need to focus, not only for the purpose of refining our terminology and definitions but also, and more importantly, to clearly determine the underlying pathogenic mechanisms of EAATD. Meanwhile, whatever the degree of uncertainty and skepticism, we must nonetheless correctly diagnose and manage the patients who present with active thyroid autoimmunity and a picture of encephalopathy, even if the concurrence of these events is rarely observed in our clinical experience and notwithstanding the ill-defined pathogenesis of this disorder. It is obvious that as long as this state of ambiguity and foot-dragging subsists, the most appropriate clinical approach to EAATD will not be achieved and the risk of under-diagnosis or inappropriate management of patients will persist.

We feel that encephalopathy, although a rare occurrence in patients with autoimmune thyroid disease, should be added to the list of other better characterized conditions such as ophthalmopathy, myopathy, and dermopathy. It is time for endocrinologists to sharpen their awareness of EAATD and collaborate closely with neurologists in order to accurately define EAATD and thereby more effectively diagnose and 
manage patients with this condition. A multidisciplinary approach could lead to significant progress in the care of patients with EAATD and in the evaluation of the epidemiology and the pathogenesis involved. It is quite possible that by increasing our awareness of this association the recorded incidence of EAATD will increase.

The authors did not receive any grant or fellowship supporting the writing of this manuscript

\section{REFERENCES}

1. Brain L, Jellinek EH, Ball K, 1966 Hashimoto's disease and encephalopathy. Lancet 2: 512-514.

2. Sawka AM, Fatourechi V, Boeve BF, Mokri B, 2002 Rarity of encephalopathy associated with autoimmune thyroiditis: a case series from Mayo Clinic from 1950 to 1996. Thyroid 12: 393-398.

3. Tamagno G, Celik Y, Simo R, et al, 2010 Encephalopathy associated with autoimmune thyroid disease in patients with Graves' disease: clinical manifestations, follow-up and outcomes. BMC Neurol 10: 27.

4. Cantón A, de Fàbregas O, Tintoré M, Mesa J, Codina A, Simó R, 2000 Encephalopathy associated to autoimmune thyroid disease: a more appropriate term for an underestimated condition? J Neurol Sci 176: 65-69.

5. Mahad DJ, Staugaitis S, Ruggieri P, et al, 2005 Steroidresponsive encephalopathy associated with autoimmune thyroiditis and primary CNS demyelination. J Neurol Sci 228: 3-5.

6. Castillo P, Woodruff B, Caselli R, et al, 2006 Steroidresponsive encephalopathy associated with autoimmune thyroiditis. Arch Neurol 63: 197-202.

7. Tamagno G, Federspil G, Murialdo G, 2006 Clinical and diagnostic aspects of encephalopathy associated with autoimmune thyroid disease (or Hashimoto's encephalopathy). Intern Emerg Med 1: 15-23.

8. Fatourechi V, 2005 Hashimoto's encephalopathy: myth or reality? An endocrinologist's perspective. Best Pract Res Clin Endocrinol Metab 19: 53-66.

9. Ferracci F, Carnevale A, 2006 The neurological disorder associated with thyroid autoimmunity. J Neurol 253: 975-984.

10. Chong J, Rowland L, Utiger R, 2003 Hashimoto encephalopathy: syndrome or myth? Arch Neurol 60: 164-171. 